\title{
Towards Vague Query Answering in Logic Programming for Logic-based Information Retrieval
}

\author{
Umberto Straccia \\ ISTI - CNR, Pisa, ITALY \\ straccia@isti.cnr.it
}

\begin{abstract}
We address a novel issue for logic programming, namely the problem of evaluating ranked top-k queries. The problem occurs for instance, when we allow queries such as "find cheap hotels close to the conference location" in which vague predicates like cheap and close occur. Vague predicates have the effect that each tuple in the answer set has now a score in $[0,1]$. We show how to compute the top-k answers in case the set of facts is huge, without evaluating all the tuples.
\end{abstract}

Keywords: Logic Programming, Fuzzy, Top-k retrieval

\section{Introduction}

In this paper we address a novel issue for Logic Programs (LPs) with a huge set of facts, namely the problem of evaluating ranked top-k queries. In classical logic programming, an answer to a query is a set of tuples that satisfy a query. Each tuple may or may not satisfy the predicates in the query. However, very often the information need of a user involves so-called fuzzy/vague predicates. For instance, a user may have the following information need: "Find cheap hotels near to the conference location". Here, cheap and near are fuzzy predicates. Unlike the classical case, tuples satisfy now these predicates to a score (usually in $[0,1]$ ). In the former case the score may depend, e.g., on the price, while in the latter case it may depend e.g. on the distance between the hotel location and the conference location.

Therefore, a major problem we have to face with in such cases is that now an answer is a set of tuples ranked according to their score. This poses a new challenge in case we have to deal with a huge amount of facts. Indeed, virtually every tuple may satisfy a query with a non-zero score and, thus, has to be ranked. Of course, computing all these scores, ranking them and then selecting the top- $k$ ones is not feasible in practice.

In this work, we address the top- $k$ retrieval problem for Datalog. At the extensional level, each fact may have a score, while at the intentional level rules describe the domain of application. Queries are conjunctive queries in which vague predicates may occur.

\section{Preliminaries}

The formalism we consider is defined as follows. The score space (i.e. truth space) is $\mathcal{S}=[0,1]$. We anticipate informally that an interpretation will assign a score (i.e. truth) 
to a ground atom and that the answers to a query (i.e. ground instances of an atom) will be ranked (in decreasing order) according to their scores.

A term is either a variable or a constant.

Let $\mathcal{V}_{E}$ and $\mathcal{V}_{I}$ be disjoint sets of $n$-ary extensional and intentional predicate symbols, respectively. An atom is of the form $p\left(t_{1}, \ldots, t_{n}\right)$, where $p$ is an $n$-ary predicate symbol and all $t_{j}$ are terms. An atom is ground if no variable occurs in it. A logic program $\mathcal{P}$ is made out of an extensional database (EDB), $\mathcal{P}_{E}$, and an intentional database (IDB), $\mathcal{P}_{I}$. The extensional database is a set of facts of the form $r(\mathbf{c}) \leftarrow b$, where $r(\mathbf{c})$ is a ground atom, $r$ is an extensional predicate and $b \in \mathcal{S}$ is a score value. The intuition here is that $b$ is the assigned score to tuple $\mathbf{c}$ in relation $r$. For convenience, for each $n$-ary extensional predicate $r$, we represent the facts $r\left(c_{1}, \ldots, c_{n}\right) \leftarrow b$ in $\mathcal{P}$ by means of a relational $n+1$-ary table $T_{r}$, containing the records $\left\langle c_{1}, \ldots, c_{n}, b\right\rangle$. Thus, the table contains all the instances of $r$ together with their scores. We assume that there cannot be two records $\left\langle c_{1}, \ldots, c_{n}, b_{1}\right\rangle$ and $\left\langle c_{1}, \ldots, c_{n}, b_{2}\right\rangle$ in $T_{r}$ with $b_{1} \neq b_{2}$ (in case their are, we remove the one with the lower score). Each table is sorted in descending order with respect to the scores. Usually, the score of a tuple in a relation has been computed (possibly off-line) by a specific system. For instance, we may have an underlying image retrieval system that for each identified object in an image has $m$ scores, one for each of the $m$ attributes (see, e.g. [8]).

The intentional database is a set of rules in which all variables in the head do also appear in the rule body. To facilitate the reading, we first give an example of rule with its intended meaning and then provide the formal definition. Assume we would like to represent the set of good conference hotels $q(x)$, which are hotels close to the conference location. Assume that we have a relational table of hotels, their location and their price, a table of conferences and their location, a distance table reporting the distance among two locations, and two tables for the extensional predicates, cheap and close, whose instances are scored with respect to the following functions, where the former depends on the price, while the latter depends on the distance: $s_{\text {cheap }}(p)=\max (0,1-p / 200)$, and $s_{\text {close }}(d)=\max (0,1-d / 2000)$. Then following rule may be a candidate rule: for a given conference $c$

$$
\begin{aligned}
q(h) \leftarrow \min [\text { hotel }(h, h L o c, \text { price }), \text { conference }(c, c L o c), \\
\text { distance }(h L o c, c L o c, d)] \cdot \operatorname{cheap}(\text { price }) \cdot \operatorname{close}(d) .
\end{aligned}
$$

Essentially, in the above rule, the score of $q(h)$ is determined by taking the min of the first three atoms and then take the product of it with the last expression. This is similar as it happens in top- $k$ retrieval in the context of relational databases $[3,4,6]$ : the data is represented in relational tables and the SQL query language is extended to allow to express a scoring function, which may use the values occurring in the retrieved records, to compute the final score of the retrieved record.

So, let $\mathcal{F}$ be a set of total score combination functions, i.e. computable functions ${ }^{1}$ $f:[0,1]^{n} \rightarrow[0,1]$ used to manipulate score values, e.g. $\min , \max , \cdot,+, \ldots$. Score combination functions will have a fixed interpretation, i.e. we will consider them as built-in functions. Then an intentional database is a set of rules of form $p(\mathbf{x}) \leftarrow f\left(A_{1}, \ldots, A_{n}\right)$, where $(i) p$ is an intentional predicate; $(i i) A_{i}$ is an atom $q(\mathbf{t})$ and $\mathbf{t}$ is a tuple of terms ( $q$ is either an intentional or an extensional predicate symbol); and (iii) $f$ is a score

\footnotetext{
${ }^{1}$ With computable we mean that for any input, the value of $f$ can be determined in finite time.
} 
combination function, which is assumed to be monotone in its arguments. Note that the extensional predicates do not occur in the head of rules of the intentional database. Essentially, we do not allow that the fact predicates occurring in $\mathcal{P}_{E}$ can be redefined by $\mathcal{P}_{I}$. A classical rule is one in which $f$ is min.

From the semantics point of view, the Herbrand universe $H_{\mathcal{P}}$ of $\mathcal{P}$ is the set of constants appearing in $\mathcal{P}$. If there is no constant symbol in $\mathcal{P}$ then consider $H_{\mathcal{P}}=\{c\}$, where $c$ is an arbitrary chosen constant. The Herbrand base $B_{\mathcal{P}}$ of $\mathcal{P}$ is the set of ground instantiations of atoms appearing in $\mathcal{P}$ (ground instantiations are obtained by replacing all variable symbols with constants of the Herbrand universe). Let $\mathcal{P}^{*}$ be the set of ground rule instantiations obtained from $\mathcal{P}$. Note that $\mathcal{P}^{*}$ is always finite. An interpretation $I$ is a partial mapping from intentional and extensional atoms to $[0,1]$ (we recall that for a constant $c, I(c)=c$ ). Note that, as $I$ may be a partial function, some atoms may not have a score. Alternatively, we may assume $I$ to be a total function. We use the former formulation to distinguish the case where a tuple $\mathbf{c}$ may be retrieved, though the score is 0 , from the tuples which do not satisfy the query and, thus, would be not retrieved. In particular, if a tuple does not belong to an extensional relation then its score is assumed to be undefined, while if $I$ is total, then the score of this tuple would be 0 . We denote with $\operatorname{def}(I)$ the set of ground atoms on which $I$ is defined. We say that $I$ is a model of $\mathcal{P}$, denoted $I \models \mathcal{P}$, iff for all facts $A \leftarrow b \in \mathcal{P}_{E}, I(A) \geq b$ and for all rules $A \leftarrow f\left(A_{1}, \ldots, A_{n}\right) \in \mathcal{P}_{I}$ such that all $I\left(A_{i}\right)$ are defined, $I(A) \geq$ $f\left(I\left(A_{1}\right), \ldots, I\left(A_{n}\right)\right)$ holds (note that the function $f \in \mathcal{F}$ has a fixed interpretation, which we identify with $f$ itself). We say that an interpretation $I$ is a minimal model of $\mathcal{P}$ iff $I=\mathcal{P}$ and for any other model $J$ of $\mathcal{P}, \operatorname{def}(I) \subseteq \operatorname{def}(J)$ and for all $A \in$ de $f(I) I(A) \leq J(A)$ holds. $^{2}$ It is not difficult to see that there is an unique minimal model $M_{\mathcal{P}}$ of $\mathcal{P}$. The proof is based on the existence of a partial monotone immediate consequence operator $T_{\mathcal{P}}$, whose fixed-points are models of $\mathcal{P}$ : for any ground atom $A \in B_{\mathcal{P}} T_{\mathcal{P}}(I)(A)=\max \left\{I(\varphi) \mid A \leftarrow \varphi \in \mathcal{P}^{*}\right\}$, where $\max \emptyset$ is undefined $\left(\mathcal{P}^{*}\right.$ is finite, so max can be used).

A query is an intentional predicate symbol $q$. The answer set of $q$ w.r.t. $\mathcal{P}$ is defined as the set $\operatorname{ans}(q, \mathcal{P})$ of tuples $\langle\mathbf{c}, s\rangle \in H_{\mathcal{P}} \times \ldots \times H_{\mathcal{P}} \times[0,1]$ such that $M_{\mathcal{P}}(q(\mathbf{c}))=s$ (the score of $\mathbf{c}$ is $s$ in the minimal model).

Example 1. Given the logic program $\mathcal{P}=\{(q(x) \leftarrow 0.5 \cdot(p(x)+r(x))),(p(a) \leftarrow$ $0.9),(p(b) \leftarrow 0.2),(r(b) \leftarrow 0.4)\}$ then $M_{\mathcal{P}}(p(a))=0.9, M_{\mathcal{P}}(p(b))=0.2, M_{\mathcal{P}}(r(b))=$ $0.4, M_{\mathcal{P}}(q(b))=0.3$ and ans $(q, \mathcal{P})=\{\langle b, 0.3\rangle\}$, while ans $(p, \mathcal{P})=\{\langle a, 0.9\rangle,\langle b, 0.2\rangle\}$. If $M_{\mathcal{P}}$ has to be a total interpretation then additionally $M_{\mathcal{P}}(r(a))=0, M_{\mathcal{P}}(q(a))=$ 0.45 and $\langle a, 0.45\rangle \in \operatorname{ans}(q, \mathcal{P})$.

Example 2. Given the logic program $\mathcal{P}=\{(q(x) \leftarrow(q(x)+1) / 2),(p(a) \leftarrow 0.4)\}$ then $M_{\mathcal{P}}(p(a))=0.4, M_{\mathcal{P}}(q(a))=1$ and ans $(q, \mathcal{P})=\{\langle a, 1\rangle\}$. Note that $\mathcal{P}$ exhibits a well-known behaviour, requiring $\omega$ steps of $T_{\mathcal{P}}$ iterations to obtain the minimal model $[5,13]$.

The basic reasoning service that mainly concerns us is:

\footnotetext{
${ }^{2}$ The least interpretation is unique and is $I_{\perp}$, where $\operatorname{def}\left(I_{\perp}\right)=\emptyset$, i.e. $I_{\perp}$ is undefined every-
} where. 
Top- $k$ retrieval: Given $\mathcal{P}$, retrieve the top- $k$ ranked tuples of the answer set of $q$ w.r.t. the score, denoted $\operatorname{ans}_{k}(q, \mathcal{P})=\operatorname{Top}_{k}(\operatorname{ans}(q, \mathcal{P}))$.

We note that retrieving the top- $k$ answers of an extensional predicate symbol $r$ is trivial as we have just to retrieve the first $k$ tuples in the relational table $T_{r}$ associated to $r$. Hence, we restrict top- $k$ retrieval to intentional predicates only.

\section{Top- $k$ information retrieval}

We next provide an incremental top-down top- $k$ query answering algorithm. Note that, as Example 2 shows, computing an answer (and, thus, the top- $k$ answers) may not be possible in finite time in general. A usual way to overcome this situation is to rely on bounded score combination functions $f$, i.e. for all $i, f\left(x_{1}, \ldots, x_{n}\right) \leq x_{i}$. In this case it can be shown that the least-fixed point is reached after a finite number of $T_{p}$ iterations [9].

To start with, we use the usual relation "directly depends on" among predicate symbols, i.e. given $\mathcal{P}$, we say that predicate symbol $p$ directly depends on predicate symbol $q$ if there is a rule in $\mathcal{P}$ such that $p$ occurs in the head of it and $q$ occurs in the body of it. The relation depends on is the transitive closure of "directly depends on". The dependency graph of $\mathcal{P}$ is a directed graph where nodes are predicate symbols and the set of edges is the "directly depends on" relation. The program is recursive if there is a cycle in the dependency graph (i.e. there is $p$ depending on $p$ ). We also say that $\mathcal{P}$ is deterministic if for each intentional predicate symbol $p$ there is at most one rule in $\mathcal{P}$ having $p$ in its head.

A practical useful case is when the logic program contains only classical rules, except for the rules having the query in the head. This depicts the scenario when a top- $k$ query involving vague predicates is issued on top of a classical logic program (deductive database), as for the "find cheap hotels" example. We call such programs classical top- $k$ programs. The top- $k$ retrieval problem for non-recursive classical top- $k$ programs has been addressed in [11], were it has been shown that for a non-recursive classical top- $k$ program $\mathcal{P}$ and query predicate $q$, $\operatorname{ans}_{k}(q, \mathcal{P})$ can be determined in LogSpace w.r.t. the size of $\mathcal{P}_{E}$.

The procedure is based on a query reformulation step, in which a rule involving the query predicate in the head is reformulated by replacing an atom $A$ in the body by means of the rule body $\phi$, for $A \leftarrow \phi \in \mathcal{P}$, and finally applying a top- $k$ algorithm for relational databases to the obtained query transformations.

For the more general case, this simple strategy is no longer possible. Of course, we always have the possibility to compute all answers (whenever termination is guaranteed), to rank them and to select the top- $k$ ones only. However, this requires to compute the score of all answers. We would like to avoid this in cases in which the extensional database is large and potentially too many tuples would satisfy the query. A distinguishing feature of our query answering procedure is that we do not determine all answers by discovering all proofs as e.g. in [5, 13], but rather apply a variant of so-called memoing techniques developed for classical logic programming -see, e.g. [15] for an overview. Essentially, the basic idea of our procedure is to collect, during the computation, all answers incrementally together in a similar way as it is done for classical Datalog. Hence, 
for instance, we do not rely on any notion of atom unification, but rather iteratively access relational tables using relational algebra.

The presentation of our algorithm proceeds as follows. We present a top- $k$ answering procedure for deterministic logic programs (at most one rule per predicate symbol $p$ in the head). Due to lack of space we are not able to include also the more general case of non-deterministic LPs as well (more than one rule per predicate symbol $p$ in the head), which will be included in an extended version of the paper. For this latter case, we just show the problem introduced and outline the solution for it. For the rest of this paper we will assume that the score combination functions are bounded, to avoid such cases as shown in Example 2.

Given $r: q(\mathbf{x}) \leftarrow \phi \in \mathcal{P}$, with $\mathbf{s}(q, r)$ we denote the set of sons of $q$ w.r.t. $r$, i.e. the set of intentional predicate symbols occurring in $\phi$. With $\mathrm{p}(q)$ we denote the set of parents of $q$, i.e. the set $\mathrm{p}(q)=\left\{p_{i}: q \in \mathrm{s}\left(p_{i}, r\right)\right\}$ (the set of predicate symbols directly depending on $q$ ).

Top- $k$ query answering for deterministic LPs. The procedure TopAnswers is detailed in Table 1. The procedure uses some auxiliary functions and data structures: $(i)$ the variable rankedList contains, for each intentional predicate $p$, the current top-ranked tuples together with their score. For each $p$, the tuples $\langle\mathbf{c}, s\rangle$ in rankedList $(p)$ are ranked in decreasing order with respect to the score $s$. We do not allow $\langle\mathbf{c}, s\rangle$ and $\left\langle\mathbf{c}, s^{\prime}\right\rangle$ to occur in rankedList $(p)$ with $s \neq s^{\prime}$ (if so, we remove the tuple with the lower score); (ii) the variable dg collects the predicate symbols the query predicate $q$ depends on; (iii) the array variable exp traces the rule bodies that have been "expanded" (the predicate symbols occurring in the rule body are put into the active list); $(i v)$ the variable in keeps track of the predicate symbols that have been put into the active list so far due to an expansion (to avoid, to put the same predicate symbol multiple times in the active list due to rule body expansion). There are other variables, which however do play a role in the procedure getNextTuple only (see Table 1), and are defined in the TopAnswers procedure as they act as global variables. We will discuss them in detail once we address the getNextTuple procedure later on.

Overall, the procedure works as follows. Assume, we are interested in determining the top- $k$ answers of $q(\mathbf{x})$. We start with putting the predicate symbol $q$ in the active list of predicate symbols A. At each iteration step we select a new predicate $p$ from the queue A and get a new tuple (getNexTuple $(p, r)$ ) satisfying the rule body $r$ whose head contains $p$ with respect to the answers gathered so far. If the evaluation leads to a new answer for $p\left(\Delta_{r} \neq \emptyset\right)$, we update the current answer set rankedList $(p)$ and add all predicates $p_{j}$ directly depending on $p$ to the queue A. At some point the active list will become empty and we have actually found correct answers of $q(\mathbf{x})$. A threshold will be used to determine when we can stop retrieving tuples. Indeed, the threshold determines when any newly retrieved tuple for $q$ scores lower than the current top- $k$ and, thus, cannot modify the top- $k$ ranking (step 9). So, step 1 loops until we do not have $k$ answers above the threshold or, two successive loops do not modify the current set of answers (step 9). Step 2 initializes the active list of predicates. Step 3. loops until no predicate has to be processed anymore. In step 4, we select a predicate symbol to be processed. In step 5, we retrieve the next answer for $p$. If a new answer has been retrieved (step 6, $\Delta_{r} \neq \emptyset$ ) then we update the current answer set rankedList $(p)$ and 
Table 1. The top- $k$ query answering procedure.
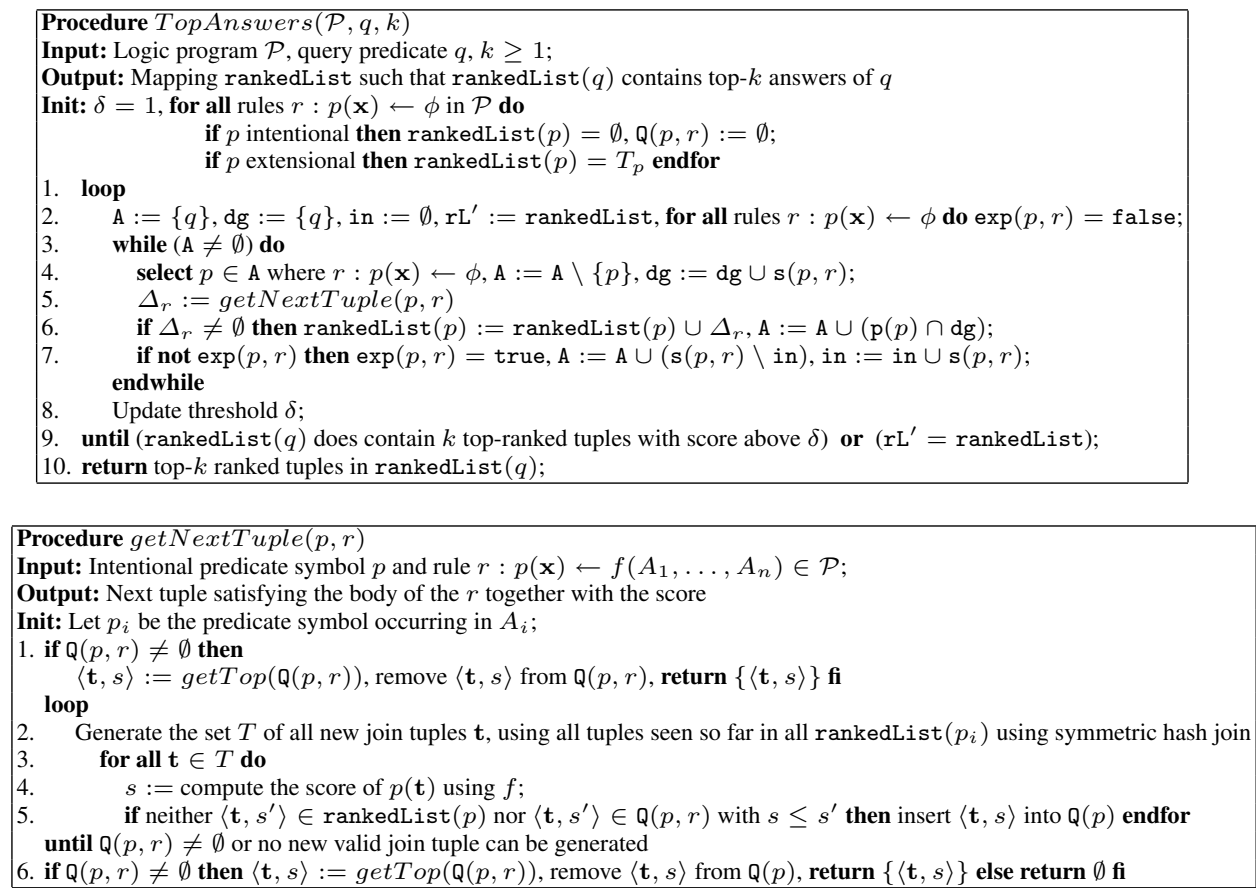

add all predicates $p_{j}$, that directly depend on $p$, to the queue A. In step 7, we put once all intentional predicate symbols appearing in the rule body of $p$ in the active list for further processing.

We next describe the getNextTuple procedure (see Table 1). It's main purpose is, given a predicate symbol $p$ and a rule $r: p(\mathbf{x}) \leftarrow \phi$, to get back the next tuple (and its score) satisfying the conditions of the rule $r$. The procedure is a generalization of the analogous get Next procedure described in [4] and uses the so-called symmetric Hash Rank Join (HRJN) algorithm. This is not surprising as the list of atoms in a rule body may be seen as multiple joins together with a scoring function.

Let us first describe the intuition behind the procedure. For the sake of illustrative purposes assume that the rule $r$ associated to $p$ is $p(x) \leftarrow p_{1}(x, y) \cdot p_{2}(y, z) \in \mathcal{P}$. The idea is as follows:

1. we incrementally generate new valid join combinations $\langle x, y, z\rangle$ from the tuples in rankedList $\left(p_{1}\right)$ and rankedList $\left(p_{2}\right)$ using some join strategy. In particular, as [4], we alternatively access first rankedList $\left(p_{1}\right)$ and then rankedList $\left(p_{2}\right)$. We select the next unseen tuple in rankedList $\left(p_{1}\right)$ and then build all join combinations with the tuples seen so far in rankedList $\left(p_{2}\right)$. Then we select the next unseen tuple in rankedList $\left(p_{2}\right)$ and then build all join combinations with the tu- 
ples seen so far in rankedList $\left(p_{1}\right)$ and so on until we find some valid join tuples $(\mathrm{Q}(p, r) \neq \emptyset)$.

2. the join tuples and their scores will be put in the queue $\mathrm{Q}(p, r)$ and the top-ranked one is returned.

Specifically, in step 1, whenever we already have some tuples in the queue $\mathrm{Q}(p, r)$ of $p$ (obtained by a previous call) then we just return the top-ranked one. Ties are split randomly. In step 2 we generate all candidate joins, involving all seen tuples of the predicates occurring in the rule body of $p$. For each of them we compute its score (step 4 ). We put the results on the queue $\mathrm{Q}(p, r)$ (step 5) and return the top-ranked one. As $\mathrm{Q}(p, r)$ may still contain answers for $p$, the next time we ask for a next tuple with respect to $p$ and $r$, we access $\mathbf{Q}(p, r)$ directly (step 1 ).

Finally, threshold $\delta$ is determined as follows. It is computed as in [4]. Suppose that for the query predicate $q$ we have a rule $r: q(x) \leftarrow f\left(p_{1}, \ldots, p_{n}\right) \in \mathcal{P}$. Let $\mathbf{t}_{i}^{\perp}$ be the last tuple seen in rankedList $\left(p_{i}\right)$, while let $\mathbf{t}_{i}^{\top}$ be the top ranked one in rankedList $\left(p_{i}\right)$. With $\mathbf{t}_{i}$.score we indicate the tuple's score. Then $\delta$ is the maximum of the following $n$ values:

$$
\begin{aligned}
\delta_{1} & =f\left(\mathbf{t}_{1}^{\perp} \cdot \text { score }, \mathbf{t}_{2}^{\top} \cdot \text { score }, \ldots, \mathbf{t}_{n}^{\top} \cdot \text { score }\right) \\
\delta_{2} & =f\left(\mathbf{t}_{1}^{\top} . \text { score }, \mathbf{t}_{2}^{\perp} \cdot \text { score }, \ldots, \mathbf{t}_{n}^{\top} \cdot \text { score }\right) \\
\vdots & \vdots \\
\delta_{n} & =f\left(\mathbf{t}_{1}^{\top} \text {.score }, \mathbf{t}_{2}^{\top} . \text { score }, \ldots, \mathbf{t}_{n}^{\perp} . \text { score }\right) .
\end{aligned}
$$

For instance, for $q(x) \leftarrow p_{1}(x, y) \cdot p_{2}(y, z) \in \mathcal{P}$ we have

$$
\begin{aligned}
\delta_{1} & =\mathbf{t}_{1}^{\perp} \text {.score } \cdot \mathbf{t}_{2}^{\top} \text {.score } \\
\delta_{2} & =\mathbf{t}_{1}^{\top} \text {.score } \cdot \mathbf{t}_{2}^{\perp} \text {.score } \\
\delta & =\max \left(\delta_{1}, \delta_{2}\right) .
\end{aligned}
$$

It is not difficult to see that whenever we consider a new join combination, its score will be below to $\delta$. Indeed, if we consider a new join tuple using the next unseen tuple from rankedList $\left(p_{1}\right)$ and a seen tuple in rankedList $\left(p_{2}\right)$, its score will be below $\delta_{1}$, while if we consider a new join tuple using the next unseen tuple from rankedList $\left(p_{2}\right)$ and a seen tuple in rankedList $\left(p_{1}\right)$, its score will be below $\delta_{2}$. Therefore, overall the score will be below $\delta$. It is thus not surprising that whenever we have top- $k$ answers for

\begin{tabular}{|c|c|c|}
\hline recId & $r_{1}$ & $r_{2}$ \\
\hline 1 & $\begin{array}{llll}a & b & 1.0 \\
\end{array}$ & $m h 0.95$ \\
\hline 2 & $\begin{array}{lll}c & d & 0.9\end{array}$ & $m j 0.85$ \\
\hline 3 & $\begin{array}{lll}e & f & 0.8 \\
\end{array}$ & $\begin{array}{lll}f & k & 0.75\end{array}$ \\
\hline 4 & $\begin{array}{llll}l & m & 0.7\end{array}$ & $m n 0.65$ \\
\hline 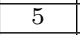 & $\begin{array}{lll}o & p & 0.6 \\
\end{array}$ & $\begin{array}{lll}p & q & 0.55 \\
\end{array}$ \\
\hline & & \\
\hline
\end{tabular}
$q$ with score above $\delta$ we can stop the retrieval process (see step 9 of TopAnswers). This property can be generalized to $n$-ary joins (see [4], Theorem 4.2.1). For the sake of illustrative purposes, let us consider the following abstract examples.

Example 3. Assume that we have the following query rule $q(x) \leftarrow \min \left(r_{1}(x, y), r_{2}(y, z)\right)$, where $q$ is the query predicate and $r_{1}, r_{2}$ are extensional predicates with tables (with millions of tuples) 
The table below reports a top-2 retrieval computation. The left table reports data related to each loop in the TopAnswers procedure, while the other shows at each iteration the execution get NextTuple $\left(r_{j}(i)\right.$ means that we access the $i$-th tuple in relation $\left.r_{j}\right)$. The first call of getNextTuple $(q)$ requires several alternative accesses to $r_{i}$ before a tuple can be found $(\langle e, k, 0.75\rangle)$. In the second call we get immediately two candidate tuples. In the third call, as $\mathrm{Q}(q, r) \neq \emptyset$ we get immediately the next candidate $(\langle l, j, 0.7\rangle)$. Finally, in the fourth call, we retrieve $\langle l, n, 0.65\rangle$. As now rankedList $(q)$ contains 2 answers above the threshold of 0.7 , we can stop and return $\{\langle e, k, 0.75\rangle,\langle l, h, 0.7\rangle\}$. Note that no new retrieved answer may have a score above 0.7 . Indeed, the next one would be $\langle o, q, 0.55\rangle$ and, thus, not all tuples are processed (which would be unfeasible in practice).

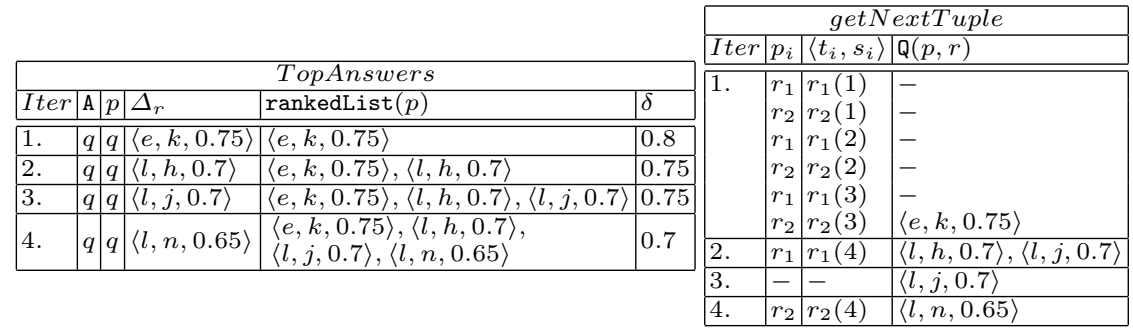

From computational point of view, by a similar analysis as in [9], it can be shown that TopAnswer is exponential with respect to $|\mathcal{P}|$ (combined complexity), but polynomial in $\left|\mathcal{P}_{E}\right|$ (data complexity), and we have:

Proposition 1. Given a deterministic logic program $\mathcal{P}$ in which all scoring functions are bounded, then TopAnswers $(\mathcal{P}, q, k)$ terminates with $\operatorname{Top} A n \operatorname{swer}(\mathcal{P}, q, k)=$ $\operatorname{ans}_{k}(q, \mathcal{P})$.

Top- $k$ query answering for general LPS. We first illustrate the problem that is introduced in the case a predicate symbol $p$ is in the head of multiple rules and then sketch how we solve it. Our top- $k$ retrieval algorithm is based on the fact that whenever we find a new instance $\langle\mathbf{c}, s\rangle$ for a predicate $p$ occurring in $\mathcal{P}$, any successive retrieved instance $\left\langle\mathbf{c}^{\prime}, s^{\prime}\right\rangle$ for $p$ is scored lower than $\langle\mathbf{c}, s\rangle$, i.e. $s^{\prime} \leq s$ (the fact that score combination functions are bounded is crucial here), which allows us to apply the stopping criteria based on a threshold. Unfortunately, if $p$ is in the head of more than one rule this is no longer true. Indeed, clearly two rules $p(\mathbf{x}) \leftarrow \phi_{1}$ and $p(\mathbf{x}) \leftarrow \phi_{2}$ are equivalent to the rule $p(\mathbf{x}) \leftarrow \max \left(\phi_{1}, \phi_{2}\right)$, and max is not a bounded score combination function. So, it is not difficult to find an example where given a retrieved instance $\langle\mathbf{c}, s\rangle$ for $p$, a successive retrieved instance $\left\langle\mathbf{c}^{\prime}, s^{\prime}\right\rangle$ for $p$ may have a score higher than $\langle\mathbf{c}, s\rangle$, i.e. $s^{\prime}>s$.

Example 4. Consider the seven rules $q(x) \leftarrow t_{1}(x), q(x) \leftarrow p_{1}(x), p_{1}(x) \leftarrow t_{2}(x)$, $t_{1}(a) \leftarrow 0.4, t_{1}(b) \leftarrow 0.3, t_{2}(c) \leftarrow 0.5, t_{2}(d) \leftarrow 0.2$. A naive extension of our procedure, may retrieve first $\langle a, 0.4\rangle$ for $q$, second $\langle c, 0.5\rangle$, third $\langle b, 0.3\rangle$ and, eventually $\langle d, 0.2\rangle$.

As we can see in the above example, it may not be guaranteed that any successive retrieved tuple for $q$ is scored lower than the previous one. However, there is still a 
simple strategy to overcome to this problem. In fact, note that any successively retrieved tuple for rule $r_{1}: q(x) \leftarrow t_{1}(x)$ is scored lower than the one retrieved before for $r_{1}$. Similarly, any successively retrieved tuple for rule $r_{2}: q(x) \leftarrow p_{1}(x)$ is scored lower than the one retrieved before for $r_{2}$. Therefore, one strategy may be to gather at least one answer for each of the rules $r_{1}$ and $r_{2}$ and only then merge the retrieved answers for $r_{1}$ and $r_{2}$ to build the answers for $q$. This will guarantee that successively retrieved answers for $r_{1}$ and $r_{2}$ are scored lower than the already retrieved ones for $q$. Of course, the threshold $\delta$ for $q$ is now $\delta=\max \left(\delta_{r_{1}}, \delta_{r_{2}}\right\}$, where $\delta_{r_{1}}$ and $\delta_{r_{2}}$ are computed as previously for rule $r_{1}$ and $r_{2}$, respectively. The detailed procedure will be described in more detail in an extended work.

\section{Conclusions}

The problem of top- $k$ retrieval will be an important problem, e.g. in logic-based (multimedia) information retrieval. We have addressed this issue in the context of logic programs. We are unaware of any other work addressing this problem for many-valued (recursive) logic programs (for non-recursive logic programs, see [11, 14]), computing the answers iteratively accessing relational tables using relational algebra (and, thus, is not resolution-based).

Major topics for future research include: $(i)$ can we refine our strategy in case the score combination functions are not bounded? (ii) How can we deal with aggregates (maybe relying on [7])? (iii) Can we apply similar ideas to other popular logical formalism, such as Description Logics (DLs) [1] (the only work we know about are [12, 10]) and their combination with LPs?

\section{References}

1. Franz Baader, Diego Calvanese, Deborah McGuinness, Daniele Nardi, and Peter F. PatelSchneider, editors. The Description Logic Handbook: Theory, Implementation, and Applications. Cambridge University Press, 2003.

2. Didier Dubois and Henri Prade. Possibility theory, probability theory and multiple-valued logics: A clarification. Annals of Mathematics and Artificial Intelligence, 32(1-4):35-66, 2001.

3. Ronald Fagin. Combining fuzzy information: an overview. SIGMOD Rec., 31(2):109-118, 2002.

4. Ihab F. Ilyas, Walid G. Aref, and Ahmed K. Elmagarmid. Supporting top-k join queries in relational databases. In VLDB-03, pages 754-765, 2003.

5. Michael Kifer and V.S. Subrahmanian. Theory of generalized annotated logic programming and its applications. Journal of Logic Programming, 12:335-367, 1992.

6. Chengkai Li, Kevin Chen-Chuan Chang, Ihab F. Ilyas, and Sumin Song. RankSQL: query algebra and optimization for relational top-k queries. In SIGMOD-05, pages 131-142, New York, NY, USA, 2005. ACM Press.

7. Chengkai Li, Kevin Chen-Chuan Chang, and Ihab F. Ilyas. Supporting ad-hoc ranking aggregates. In SIGMOD-06, USA, 2006. ACM Press.

8. Carlo Meghini, Fabrizio Sebastiani, and Umberto Straccia. A model of multimedia information retrieval. Journal of the ACM, 48(5):909-970, 2001.

9. Umberto Straccia. Query answering in normal logic programs under uncertainty. In ECSQARU-05, LNCS 3571, pages 687-700, Barcelona, Spain, 2005. Springer Verlag. 
10. Umberto Straccia. Answering vague queries in fuzzy DL-Lite. In Proceedings of the 11th International Conference on Information Processing and Management of Uncertainty in Knowledge-Based Systems, (IPMU-06), 2006.

11. Umberto Straccia. Towards top-k query answering in deductive databases. In Proceedings of the 2006 IEEE International Conference on Systems, Man and Cybernetics (SMC-06). IEEE, 2006.

12. Umberto Straccia. Towards top-k query answering in description logics: the case of DL-Lite. In JELIA-06, LNCS 4160, 2006. Springer Verlag.

13. Peter Vojtáš. Fuzzy logic programming. Fuzzy Sets and Systems, 124:361-370, 2001.

14. Peter Vojtáš. Fuzzy logic aggregation for semantic web search for the best (top- $k$ ) answer. In Elie Sanchez, editor, Fuzzy Logic and the Semantic Web, Capturing Intelligence, chapter 17, pages 341-359. Elsevier, 2006.

15. David S. Warren. Memoing for logic programs. Commun. ACM, 35(3):93-111, 1992. 